\title{
Optogenetics in epilepsy
}

\author{
J. Nicole Bentley, M.D., ${ }^{1}$ Cindy Chestek, Ph.D., ${ }^{2}$ William C. Stacey, M.D., Ph.D., ${ }^{3}$ \\ and Parag G. Patil, M.D., Ph.D. ${ }^{1-3}$
}

Departments of ${ }^{1}$ Neurosurgery, ${ }^{2}$ Biomedical Engineering, and ${ }^{3}$ Neurology, University of Michigan, Ann Arbor, Michigan

\begin{abstract}
Optogenetics, the use of light to stimulate or inhibit neural circuits via viral transduction of protein channels, has emerged as a possible method of treating epilepsy. By introducing viral vectors carrying algal-derived cation or anion channels, known as opsins, neurons that initiate or propagate seizures may be silenced. To date, studies using this technique have been performed in animal models, and current efforts are moving toward more sophisticated nonhuman primate models. In this paper, the authors present a brief overview of the development of optogenetics and review recent studies investigating optogenetic modification of circuits involved in seizures. Further developments in the field are explored, with an emphasis on how optogenetics may influence future neurosurgical interventions. (http://thejns.org/doi/abs/10.3171/2013.3.FOCUS1364)
\end{abstract}

\section{KEY WoRds • optogenetics • epilepsy • channelrhodopsin • halorhodopsin}

$\mathrm{E}$ PILEPSY affects more than 50 million people worldwide, or roughly $1 \%$ of the population, with a frequency of 4 to 10 per 1000 people per year. ${ }^{21,27}$ Treatment with antiepileptic drugs is frequently associated with poorly tolerated side effects, and the disease has proven to be medically refractory in more than $20 \%$ of patients. ${ }^{15}$ Resection of portions of the temporal lobe, amygdala, and hippocampus has been found to be an excellent option in the small fraction of patients suffering from temporal lobe seizures, with $86 \%-90 \%$ of patients experiencing freedom from seizures at 2 years. ${ }^{26}$ However, not all patients experience seizures emanating from the mesial temporal lobe, and of those that do, not all are candidates for resection. Therefore, there is a continued need for therapies that address focal epilepsies currently not amenable to existing techniques.

There are two general approaches to controlling seizures. The first approach, which is the basis of most antiepileptic medications, is to alter the excitability of the neural network to prevent seizures from ever occurring. This pharmacological approach has led to many new antiepileptic drugs over the past 5 decades, but the rate of intractable seizures has remained stable. ${ }^{15} \mathrm{~A}$ second approach for those intractable seizures is to abort them just as they begin, which has led to a search for medical devices that can stop seizures on demand. ${ }^{28}$ These "closedloop" devices, using a strategy similar to implanted cardi-

Abbreviations used in this paper: $\mathrm{EEG}=$ electroencephalography; GABA $=\gamma$-aminobutyric acid. ac defibrillators, would confirm the presence of a seizure and respond within milliseconds of onset, correcting the aberrant neurons while leaving normal neurons unaffected. Closed-loop devices have the advantage of limiting the intervention to specific times, which conserves power and leaves the normal neuronal or glial physiology unaltered during interictal periods. In the last decade, electrical stimulation of seizure foci via subdural or depth electrodes has gained attention. Two recent multicenter, double-blind, randomized controlled trials showed significantly reduced seizure frequencies when stimulation was applied. While the results are promising, perhaps offering a novel treatment modality for epilepsy, electrical stimulation lacks the precision of cell-type specificity. ${ }^{8}$

Optogenetics is the ability to use optical systems for the stimulation or inhibition of a specific subset of neurons by introduction of genes for light-gated channels. This is a powerful tool to turn on and off circuits of interest and has been applied to a variety of neurological and some nonneurological diseases, including investigation of myocyte physiology. ${ }^{5,7,16,35}$ In this paper we present a review of the recent literature regarding optogenetic techniques and its application to epilepsy.

\section{Optogenetic Tools}

Optogenetics is a novel molecular intervention that relies on naturally occurring opsins, or light-gated ion channels, first identified in the early 1970s. The ion channel bacteriorhodopsin, found in the plasma membrane of 


\section{J. N. Bentley et al.}

Halobacterium halobium, undergoes a conformational change in response to the absorption of light of a specific wavelength, resulting in the outflow of protons. This efflux of ions generates a life-sustaining transmembrane gradient and electric potential that the bacterial cell then uses to drive adenosine 5'-triphosphate synthesis. ${ }^{23}$ At a time when the intricacies of the chemiosmotic theory were being clarified, opsins provided insight into a prevalent biochemical mechanism. However, these channels would not be applied to neuroscience until three decades later. In 2004, researchers effectively incorporated a proton channel (channelrhodopsin-1) and a cation channel (channelrhodopsin-2) into mammalian neurons. These opsins responded to flashes of light within milliseconds, resulting in neuronal depolarization in a precise manner. ${ }^{2,19}$ Genetic engineering improved upon several aspects of the channels, with increases in photocurrent amplitudes, protein expression, and action potential reliability. Additionally, opsins were identified that were able to permit depolarizing inflow of ions in a "red-shifted" spectrum, important for neural application, as red light has increased penetrance in brain tissue. ${ }^{11}$

With the ability to turn on circuits, efforts were shifted to identification of a channel to inhibit them, which was achieved with a hyperpolarizing opsin from the halophilic archaebacterium Natronomonas pharaonis. This pump, known as halorhodopsin, a heptahelical membrane protein, converts all-trans retinal to 13-cis retinal, exchanging 1 photon for 1 chloride ion. ${ }^{12}$ The addition of a C-terminal endoplasmic reticulum export motif onto the halorhodopsin protein resulted in improved plasma membrane localization, ${ }^{10}$ and further optimization increased the photocurrent amplitude. This version of the channel, termed eNpHR3.0, has been shown to be safely and reliably expressed in freely moving animals. ${ }^{31,33}$ Continued modifications have led to a variety of engineered opsins, and recently, a tandem construct has been developed that will enable expression of both an excitatory channelrhodopsin and inhibitory halorhodopsin in 1e vector. ${ }^{29}$ The number of available opsins continues to grow and will likely continue to be optimized for experimental and human application. Figure 1 depicts overall function of these channels. ${ }^{36}$

Opsins are delivered to cells of interest by a variety of methods. Currently, injection of viral vectors into various regions may be most applicable to clinical scenarios. Subsets of cells within a region are selected using celltype specific promoters, and several of these cell types are involved in epilepsy. The opsin genes themselves are carried in viral vectors, typically adenoassociated virus or lentivirus. ${ }^{25}$ Another method of introduction involves crossing Cre-recombinase knockin animals expressing Cre in specific neurons with animals transfected with the gene of interest. This results in expression of the opsin channel only in those neurons expressing Cre-recombinase in the cell type (for review, see Madisen et al. ${ }^{17}$ ). An example of the gene is shown in Fig. 2, including the opsin gene itself and the regions needed for effective translocation. ${ }^{18}$

Light delivery to activate the opsins is another area of research, with many different systems undergoing tri- als. Thin, cylindrical "optrodes" with a tip containing an optical fiber and recording electrode are reminiscent of deep brain stimulation electrodes. ${ }^{32}$ Other versions use an optrode implanted in 1 area, with a separate micro-LED array for recording. Before human use, these systems will need to meet the standards currently used for other implantable devices, with modifications made for patient tolerance and safety.

\section{Optogenetics in Epilepsy}

Optogenetics has now been applied to numerous neurological diseases, $, 5,16,35$ as well as some nonneurological applications, including stimulation of heart muscle. ${ }^{1,3}$ Other cell types being targeted include medium spiny neurons in the basal ganglia for studying Parkinson disease,,$^{13}$ midbrain ventral tegmental area dopaminergic neurons in depression models, ${ }^{4}$ and dopamine D1-expressing neurons of the prefrontal cortex to investigate a circuit underlying temporal control of behavior. ${ }^{20}$ Probing various physiological mechanisms has also become a focus of optogenetic manipulation, with recent studies elucidating a role of ventral surface astrocytes in control of breathing. ${ }^{9}$ In epilepsy, optogenetics has become an attractive option to silence hyperactive neurons, with targeting of those believed to initiate or propagate seizures.

Epilepsy presents a difficult entity to study because of the large number of network pathologies that initiate or propagate seizures. However, there are several recent studies that aim to leverage the selectivity of optogenetics to analyze and control network dynamics in several animal models of seizures. Epilepsy is characterized by overactivity of various circuits, ${ }^{22}$ and consequently, many of the current investigations have focused on ways to silence or inhibit overactive circuits. The ability to selectively target mammalian hippocampal neurons was a first step toward probing this disease pathway, and was achieved using the inhibitory opsin halorhodopsin in organotypic cultures of mouse hippocampi. ${ }^{37}$ Following this achievement, halorhodopsin was used in selected principal glutamatergic neurons in the hippocampus. ${ }^{30}$ In this mouse model, the injection of a lentiviral vector into CA1 and CA3 pyramidal cells resulted in hyperpolarization in response to orange light (wavelength 573-613 nm). Action potentials were attenuated and paroxysmal depolarizing shifts were curbed, suppressing epileptiform activity. Importantly, the presence of the virally transfected opsins into neurons did not affect normal physiological properties of cells. To test this, organotypic hippocampal slices were subjected to complete darkness, and various properties of the cells were tested. Parameters including input resistance, resting membrane potential, action potential threshold and duration, amplitudes in response to hyperpolarizing and depolarizing pulses, and accommodation of cells to action potentials were similar in the halorhodopsin neurons in comparison with the controls.

Recently, this technology has been demonstrated in 3 different in vivo rodent models of epilepsy. In the rat tetanus toxin injection model, principal neurons were transfected with a lentiviral vector carrying halorhodopsin 2.0. Illuminating the transfected cells with 561- 


\section{Optogenetics in epilepsy}

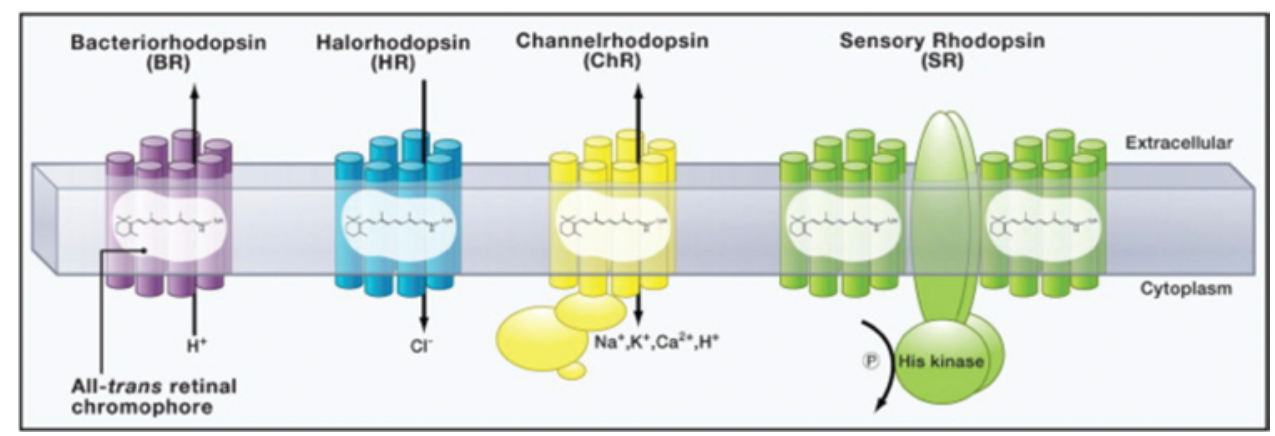

FIG. 1. Type I microbial rhodopsins. Bacteriorhodopsins (BRs) and proteorhodopsins pump protons from the cytoplasm to the extracellular medium, and halorhodopsins (HRs) pump chloride into the cytoplasm; all 3 hyperpolarize the cell. Sensory rhodopsins (SRs) lack transmembrane ion transport in the presence of the His (histidine) kinase transducer protein Htr, and algal channelrhodopsins (ChRs) conduct cations across the membrane in both directions but always along the electrochemical gradient of the transported ions. In SRs and ChRs, proton translocation within the protein is linked to efficient photocycle progression, but these protons are not necessarily exchanged between the intra- and extracellular spaces. Reprinted from Cell 147: Zhang F, Vierock J, Yizhar O, et al., The microbial opsin family of optogenetic tools, pp. 1446-1457, 2011, with permission from Elsevier.

nm wavelength light resulted in decreased epileptiform EEG activity. Decreased high-frequency power was also observed, which correlate to bursts of activity in human epilepsy, and event frequency also diminished. ${ }^{34}$

The other two examples used a closed-loop strategy to abort seizures. In the first, a cortical stroke model was used in rats to promote seizures induced by photothrombosis, in which animals were first sensitized by injection of Rose Bengal dye, after which illumination resulted in increased light absorption and focal thrombosis. Intracranial EEG electrodes confirmed spontaneous epileptiform oscillations in the thalamus and cortex of injured rats but not in controls, correlating with thalamic network hyperexcitability. To determine whether these hyperactive thalamic cells could respond to light and silence seizure activity, a viral vector was injected consisting of the cell-type specific promoter Camk2a, the halorhodopsin eNpHR3.0, optimized with membrane trafficking signal motifs, and a reporter (eYFP, yellow fluorescent protein). The promoter ensured that only excitatory thalamic cells would be subject to viral transduction. Injections were performed stereotactically into the ventrobasal thalamus, followed by slice preparation for in vitro whole-cell recordings, as well as chronic recordings and stimulation in freely moving animals. To perform the chronic recordings, a device containing multiple EEG electrodes and chronic multisite optrode was implanted into the injected thalamus and peristroke cortex. Yellow light of a wavelength $594 \mathrm{~nm}$ was delivered, which

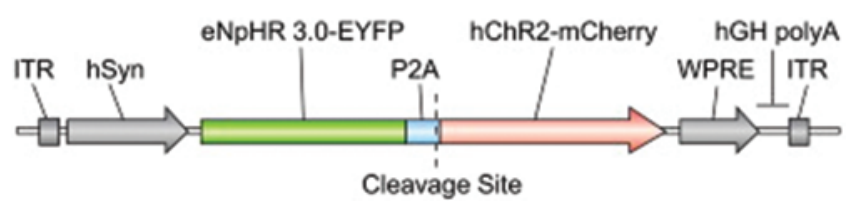

FIG. 2. A bicistronic vector used to express channelrhodopsin-2 (ChR2) and halorhodopsin (eNpHR3.0) in the same neuron. The 2 opsin genes are linked using a $2 \mathrm{~A}$ self-cleavage linker. ITR = inverted terminal repeat; hSyn = human synapsin I promoter; P2A = peptide 2A; mCherry = monomeric Cherry; hGH polyA = human growth hormone poly adenosine; WPRE = woodchuck hepatitis posttranscriptional regulatory element. Reprinted from Biol Psychiatry 71: Mei Y, Zhang F, Molecular tools and approaches for optogenetics, pp. 1033-1038, 2012, with permission from Elsevier. resulted in silencing of epileptic EEG activity in thalamus and cortex and correlated with abortion of behavioral seizures as well. Lower intensity light was not effective, and the 594-nm light did not affect interictal EEG tracings or behaviors. The authors also developed a method of detecting and interrupting seizures online by routing to a real-time processor capable of determining seizure onset, which triggered light stimulation and resulted in seizure abortion within 1 second. ${ }^{24}$

Following this report, optogenetic silencing of seizures was tested in a model of temporal lobe epilepsy. Here, mice were injected with kainic acid unilaterally into the dorsal hippocampus, and 2 methods of seizure control were explored: inhibition of excitatory principal cells and stimulation of inhibitory GABAergic cells. Opsins were expressed by using transgenic animals, crossing mice expressing the Cre-Iox recombinase in either principal cells or parvalbumin-containing GABAergic interneurons with those expressing halorhodopsin or channelrhodopsin-2, respectively. With this method, only cells containing Cre were able to express the opsin of interest, ensuring specificity. As in previous studies, a reporter fluorescent protein was also expressed. Seizure detection was achieved via depth electrodes and tuning of seizure "signatures" (magnitude, amplitude, width, rate, and frequency) with custom software. For inhibition of principal neurons with halorhodopsin, amber light of wavelength $589 \mathrm{~nm}$ was delivered via an optical fiber implanted in the ipsilateral hippocampus upon seizure activity detection. All animals $(n=6)$ responded with silencing of electrographic seizures, with a majority $(57 \% \pm 12 \%)$ aborted within 1 second. The percentage of seizures aborting within 5 seconds was approximately $70 \%$, and was unaffected by light duration (30 seconds vs 10 seconds). Further interrogation was made into the effect of red-shifted light, which has increased tissue penetrance, but is suboptimal for channelrhodopsin-2. This $635-\mathrm{nm}$ light aborted $46 \% \pm 12 \%$ seizures within 1 second of illumination. One animal in particular responded with $100 \%$ of seizures aborting within 1 second. ${ }^{14}$ The results from this portion of the experiment are shown in Fig. 3.

Activation of parvalbumin-GABAergic neurons us- 
a b
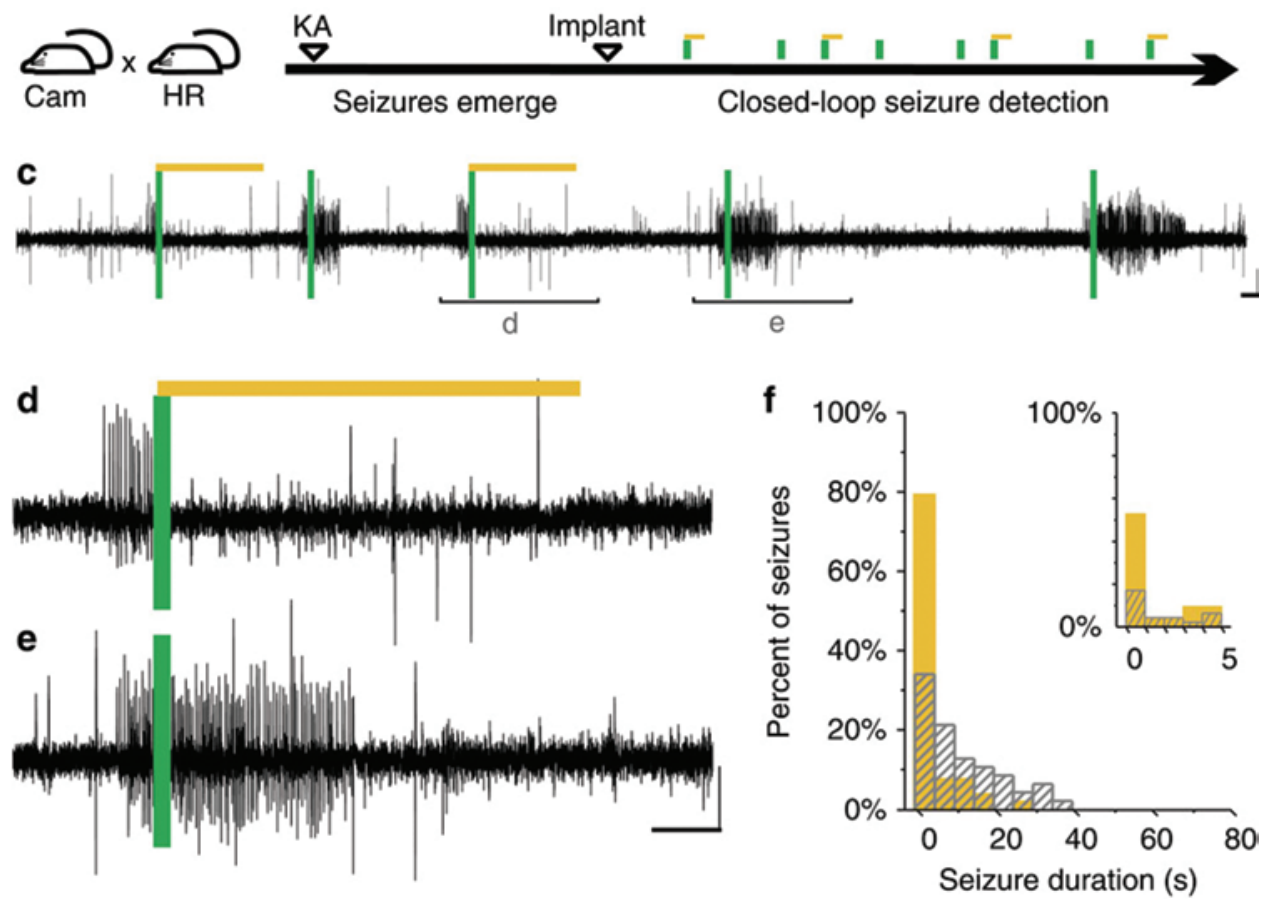

FIG. 3. Seizure control in mice expressing halorhodopsin (HR) in principal cells in a model of temporal lobe epilepsy. a: Crossing CamK-Cre and Cre-dependent HR mouse lines generated mice expressing the inhibitory opsin HR in excitatory cells (Cam-HR mice). b: Experimental timeline. c-e: Example electrographic seizures detected (vertical green bars), activating amber light $(589 \mathrm{~nm})$ randomly for $50 \%$ of events (light: amber line, example in d; no light example in e). f: Typical example distribution of postdetection seizure durations (5-second bin size) during light (solid amber) and no-light internal control conditions (hatched gray). Inset: First 5-second bin expanded, 1-second bin size. Note that most seizures stop within 1 second of light delivery. Adapted from Nat Commun 4: Krook-Magnuson E, Armstrong C, Oijala M, Soltesz I, On-demand optogenetic control of spontaneous seizures in temporal lobe epilepsy, p. 1376, 2013, with permission from Elsevier. KA = kainic acid.

ing channelrhodopsin-2 also resulted in reduced seizure duration. Blue light delivery (wavelength $473 \mathrm{~nm}$ ) aborted $59 \% \pm 11 \%$ of seizures within 5 seconds. This was a significant finding, as these cells make up less than $5 \%$ of hippocampal neurons, suggesting that even in a small population of targeted cells, optical stimulation of transgenic cells may be efficacious. ${ }^{14}$

\section{Future Directions}

These studies form the basis of our understanding of how to use light-activated channels as a therapeutic option for seizure control. Much remains to be learned from the use of opsins and light delivery, and many technical aspects must still be optimized before application to humans, several of which may prove to be substantial challenges. Among these aspects are investigation of the human immune response to these foreign proteins, the stability of viral vectors in neural tissue, and optimization of light delivery with chronically implanted devices. Thus far, application of this powerful tool has been successful in elucidating normal and pathological mechanisms, and some inquiry has been made into therapeutic applications, such as those presented here concerning epilepsy. The development of a nonhuman primate model is one recent achievement that may increase understanding of how optogenetics may be applied to humans. ${ }^{6}$

\section{Conclusions}

Epilepsy is a prevalent disease conferring a significant burden to the people affected. Despite best pharmacological treatment, medical therapies leave more than $20 \%$ of patients with refractory disease. Resection of a seizure focus is one alternative treatment, but with the large number of patients in whom surgery is not an option, there continues to be a need for novel therapies. Recent progress has been made in the field of neurostimulation via subdural or depth electrodes, but efficacy has not yet been definitively borne out in clinical trials. One limitation to these previous therapies is the inability to precisely target aberrant cells and circuits while leaving others unaltered. For this reason, optogenetics has emerged as a possible technique, and several recent studies have investigated its usefulness in animal models. Although still in the early stages, introduction of light-gated ion channels into neurons via transgenic methods has shown to be possible. Light delivery to the region of interest, with the resultant opening and closing of these channels, leading to depolarization or hyperpolarization, has also been shown in numerous studies. Much remains to be understood before application to humans, including optimization of opsin introduction, light delivery, and device implantation. Furthermore, the safety of these channels will need to be determined. However, the ability to specifically alter 
a defined circuit in a reversible but reliable manner may provide insight into the mechanism of epilepsy as well as a possible therapy.

\section{Disclosure}

The authors report no conflict of interest concerning the materials or methods used in this study or the findings specified in this paper.

Author contributions to the study and manuscript preparation include the following. Conception and design: Patil, Bentley. Acquisition of data: Bentley. Analysis and interpretation of data: Bentley. Drafting the article: Bentley. Critically revising the article: all authors. Reviewed submitted version of manuscript: all authors.

\section{References}

1. Arrenberg AB, Stainier DY, Baier H, Huisken J: Optogenetic control of cardiac function. Science 330:971-974, 2010

2. Boyden ES, Zhang F, Bamberg E, Nagel G, Deisseroth K: Millisecond-timescale, genetically targeted optical control of neural activity. Nat Neurosci 8:1263-1268, 2005

3. Bruegmann T, Malan D, Hesse M, Beiert T, Fuegemann CJ, Fleischmann BK, et al: Optogenetic control of heart muscle in vitro and in vivo. Nat Methods 7:897-900, 2010

4. Chaudhury D, Walsh JJ, Friedman AK, Juarez B, Ku SM, Koo JW, et al: Rapid regulation of depression-related behaviours by control of midbrain dopamine neurons. Nature 493:532536, 2013

5. Deisseroth K: Optogenetics and psychiatry: applications, challenges, and opportunities. Biol Psychiatry 71:1030-1032, 2012

6. Diester I, Kaufman MT, Mogri M, Pashaie R, Goo W, Yizhar O, et al: An optogenetic toolbox designed for primates. Nat Neurosci 14:387-397, 2011

7. Fiala A, Suska A, Schlüter OM: Optogenetic approaches in neuroscience. Curr Biol 20:R897-R903, 2010

8. Fridley J, Thomas JG, Navarro JC, Yoshor D: Brain stimulation for the treatment of epilepsy. Neurosurg Focus 32(3):E13, 2012

9. Gourine AV, Kasymov V, Marina N, Tang F, Figueiredo MF, Lane S, et al: Astrocytes control breathing through $\mathrm{pH}$-dependent release of ATP. Science 329:571-575, 2010

10. Gradinaru V, Thompson KR, Deisseroth K: eNpHR: a Natronomonas halorhodopsin enhanced for optogenetic applications. Brain Cell Biol 36:129-139, 2008

11. Kokaia M, Andersson M, Ledri M: An optogenetic approach in epilepsy. Neuropharmacology 69:89-95, 2013

12. Kolbe M, Besir H, Essen LO, Oesterhelt D: Structure of the light-driven chloride pump halorhodopsin at 1.8 A resolution. Science 288:1390-1396, 2000

13. Kravitz AV, Freeze BS, Parker PR, Kay K, Thwin MT, Deisseroth K, et al: Regulation of parkinsonian motor behaviours by optogenetic control of basal ganglia circuitry. Nature 466: 622-626, 2010

14. Krook-Magnuson E, Armstrong C, Oijala M, Soltesz I: On-demand optogenetic control of spontaneous seizures in temporal lobe epilepsy. Nat Commun 4:1376, 2013

15. Kwan P, Schachter SC, Brodie MJ: Drug-resistant epilepsy. N Engl J Med 365:919-926, 2011

16. Lin SC, Deisseroth K, Henderson JM: Optogenetics: background and concepts for neurosurgery. Neurosurgery 69:1-3, 2011

17. Madisen L, Mao T, Koch H, Zhuo JM, Berenyi A, Fujisawa $\mathrm{S}$, et al: A toolbox of Cre-dependent optogenetic transgenic mice for light-induced activation and silencing. Nat Neurosci 15:793-802, 2012

18. Mei Y, Zhang F: Molecular tools and approaches for optogenetics. Biol Psychiatry 71:1033-1038, 2012
19. Nagel G, Ollig D, Fuhrmann M, Kateriya S, Musti AM, Bamberg E, et al: Channelrhodopsin-1: a light-gated proton channel in green algae. Science 296:2395-2398, 2002

20. Narayanan NS, Land BB, Solder JE, Deisseroth K, DiLeone RJ: Prefrontal D1 dopamine signaling is required for temporal control. Proc Natl Acad Sci U S A 109:20726-20731, 2012

21. Ngugi AK, Bottomley C, Kleinschmidt I, Sander JW, Newton CR: Estimation of the burden of active and life-time epilepsy: a meta-analytic approach. Epilepsia 51:883-890, 2010

22. Noebels JL, Avoli M, Rogawski MA, Olsen RW, Delgado-Escueta AV (eds): Jasper's Basic Mechanisms of the Epilepsies, ed 4. New York: Oxford University Press, 2012

23. Oesterhelt D, Stoeckenius W: Functions of a new photoreceptor membrane. Proc Natl Acad Sci U S A 70:2853-2857, 1973

24. Paz JT, Davidson TJ, Frechette ES, Delord B, Parada I, Peng $\mathrm{K}$, et al: Closed-loop optogenetic control of thalamus as a tool for interrupting seizures after cortical injury. Nat Neurosci 16:64-70, 2013

25. Rein ML, Deussing JM: The optogenetic (r)evolution. Mol Genet Genomics 287:95-109, 2012

26. Sagher O, Thawani JP, Etame AB, Gomez-Hassan DM: Seizure outcomes and mesial resection volumes following selective amygdalohippocampectomy and temporal lobectomy. Neurosurg Focus 32(3):E8, 2012

27. Sørensen AT, Kokaia M: Novel approaches to epilepsy treatment. Epilepsia 54:1-10, 2013

28. Stacey WC, Litt B: Technology insight: neuroengineering and epilepsy-designing devices for seizure control. Nat Clin Pract Neurol 4:190-201, 2008

29. Tang W, Ehrlich I, Wolff SB, Michalski AM, Wölfl S, Hasan MT, et al: Faithful expression of multiple proteins via 2Apeptide self-processing: a versatile and reliable method for manipulating brain circuits. J Neurosci 29:8621-8629, 2009

30. Tønnesen J, Sørensen AT, Deisseroth K, Lundberg C, Kokaia M: Optogenetic control of epileptiform activity. Proc Natl Acad Sci U S A 106:12162-12167, 2009

31. Tye KM, Prakash R, Kim SY, Fenno LE, Grosenick L, Zarabi $\mathrm{H}$, et al: Amygdala circuitry mediating reversible and bidirectional control of anxiety. Nature 471:358-362, 2011

32. Wang J, Wagner F, Borton DA, Zhang J, Ozden I, Burwell RD, et al: Integrated device for combined optical neuromodulation and electrical recording for chronic in vivo applications. J Neural Eng 9:016001, 2012

33. Witten IB, Lin SC, Brodsky M, Prakash R, Diester I, Anikeeva P, et al: Cholinergic interneurons control local circuit activity and cocaine conditioning. Science 330:1677-1681, 2010

34. Wykes RC, Heeroma JH, Mantoan L, Zheng K, MacDonald DC, Deisseroth K, et al: Optogenetic and potassium channel gene therapy in a rodent model of focal neocortical epilepsy. Sci Transl Med 4:161ra152, 2012

35. Yizhar O, Fenno LE, Davidson TJ, Mogri M, Deisseroth K: Optogenetics in neural systems. Neuron 71:9-34, 2011

36. Zhang F, Vierock J, Yizhar O, Fenno LE, Tsunoda S, Kianianmomeni A, et al: The microbial opsin family of optogenetic tools. Cell 147:1446-1457, 2011

37. Zhang F, Wang LP, Brauner M, Liewald JF, Kay K, Watzke N, et al: Multimodal fast optical interrogation of neural circuitry. Nature 446:633-639, 2007

Manuscript submitted February 15, 2013.

Accepted March 8, 2013.

Please include this information when citing this paper: DOI: 10.3171/2013.3.FOCUS1364.

Address correspondence to: Parag G. Patil, M.D., Ph.D., Department of Neurosurgery, University of Michigan, Taubman Health Care Center, Rm 3552, 1500 East Medical Center Drive, Ann Arbor, Michigan 48109-5338. email: pgpatil@med.umich.edu. 